\title{
Fardeau économique lié aux traumatismes de la moelle épinière au Canada
}

\author{
H. Krueger, Ph. D. (1, 2); V. K. Noonan, Ph. D., PT (1, 3); L. M. Trenaman, B. Sc. (2); P.Joshi, Ph. D. (3); C. S. Rivers, Ph. D. (3)
}

Cet article a fait l'objet d'une évaluation par les pairs.

\section{Résumé}

Introduction : Cette étude vise à mesurer, au moyen d'une approche fondée sur l'incidence, le fardeau économique à vie lié aux traumatismes de la moelle épinière (TME) au Canada du point de vue social, en incluant les coûts directs et indirects.

Méthodologie : Les ressources disponibles et l'information sur les coûts liés aux cas de tétraplégie et de paraplégie complètes et incomplètes ont été appliquées à l'incidence annuelle estimative des TME au Canada en fonction de leur gravité.

Résultats : Le fardeau économique à vie d'une personne atteinte d'un TME varie de 1,5 million de dollars pour une paraplégie incomplète à 3 millions de dollars pour une tétraplégie complète. Le fardeau économique annuel lié aux 1389 nouveaux cas de TME où le patient a survécu à son hospitalisation est estimé à 2,67 milliards de dollars.

Conclusion : Malgré leur fréquence annuelle relativement faible au Canada, ces blessures entraînent un fardeau économique annuel important.

Mots-clés : traumatisme de la moelle épinière, fardeau économique, morbidité, mortalité

\section{Introduction}

Les traumatismes de la moelle épinière (TME) représentent un lourd fardeau pour les personnes atteintes, leur famille, leurs soignants et la société en général. Ces blessures entraînent, en plus de la souffrance physique et psychosociale, un lourd fardeau économique lié aux coûts accrus des soins de santé et aux taux élevés de morbidité et de mortalité précoce. Les données sur ce fardeau économique à vie lié à un TME sont cependant limitées, surtout au Canada.

La littérature existante sur les TME a tendance à se concentrer sur certains sous-groupes de patients (p. ex. anciens combattants $^{1,2}$, patients admis dans des hôpitaux utilisant les Spinal Cord Injuries Model Systems [systèmes modèles con- cernant les traumatismes de la moelle épinière] des États-Unis ${ }^{3,4,5}$ et accidents de travail $^{6}$ ), un aspect particulier du fardeau économique (p. ex. réadaptation et hospitalisation imprévues ${ }^{7,8}$ ) ou une période spécifique suivant la survenue de la blessure ${ }^{9}$.

Deux groupes de recherche - l'un au Canada $^{9,10,11}$ et l'autre aux États-Unis ${ }^{12,13}$ - ont suivi une approche fondée sur la population. Pour l'étude menée en Alberta, des données sur les coûts directs ont été recueillies pendant les six premières années suivant le traumatisme $e^{9,10,11}$. Pour sa part, l'étude américaine a tenu compte des coûts directs et indirects assumés tout au long de la vie, mais ces données datent de 1988 et ont besoin d'une mise à jour. Les coûts directs comprennent habituellement les dépenses assumées par le sys- tème de santé et par le patient ou les soignants relativement à ces blessures $^{12,13}$. Les coûts indirects " se rapportent à la valeur des pertes en matière de productivité potentielle résultant d'une réduction ou d'un arrêt du travail ou d'autres activités à la suite d'un TME. Ces coûts sont considérés comme des pertes entraînées par la réduction de la productivité résultant de la morbidité ou de la mortalité attribuable au TME » [traduction] $^{12, \text { p. } 12}$.

Dans les deux études, on a constaté qu'une approche fondée sur la population donnait lieu à un ensemble de patients très différent de celui obtenu au moyen d'une approche fondée sur des établissements $^{10,12}$. Autrement dit, les personnes atteintes d'un TME soignées par l'intermédiaire du système modèle des ÉtatsUnis, par exemple, ont tendance à avoir subi des blessures beaucoup plus graves que les patients victimes de TME au sein de la population générale ${ }^{12}$. Cette différence peut avoir des effets importants sur l'estimation du fardeau économique lié aux TME.

Par ailleurs, étant donné que la majorité de la littérature existante provient des États-Unis et que les différences entre les systèmes de soins de santé américain et canadien rendent les comparaisons difficiles, les coûts doivent être étudiés d'un point de vue canadien.

Cette étude s'appuie sur les meilleures données trouvées dans la littérature existante pour modéliser le fardeau économique à long terme actuel lié aux TME au Canada dans une perspective sociétale.

Rattachement des auteurs :

1. Université de la Colombie-Britannique, Vancouver (Colombie-Britannique), Canada

2. H. Krueger \& Associates Inc., Delta (Colombie-Britannique), Canada

3. Institut Rick Hansen, Vancouver (Colombie-Britannique), Canada

Correspondance : Carly Rivers, 6400 Blusson Spinal Cord Centre, 818 West 10th Avenue, Vancouver (Colombie-Britannique) V5Z 1M9; tél. : 604-707-2171; téléc. : 604-707-2121; courriel : crivers@rickhanseninstitute.org 


\section{Méthodologie}

Nous avons utilisé les données provenant de diverses publications pour établir notre modèle de fardeau économique à long terme lié aux TME en fonction de la gravité de la blessure et, dans la mesure du possible, nous avons utilisé des données d'études fondées sur la population canadienne $e^{9,10,11}$. Celles-ci reposaient en grande partie sur les données de deux études fondées sur la population américaine, l'une publiée en $1992^{12,13}$ et l'autre en $1998^{14}$ (tableau 1). Les coûts établis à l'aide de l'étude canadienne ont été ajustés en fonction de la valeur du dollar en 2011, selon la composante "santé et soins personnels » de l'Indice des prix à la consommation (IPC) $)^{15}$. Les coûts aux États-Unis ont été ajustés en dollars canadiens (2011) en deux temps : conversion en dollars canadiens pour l'année en question, puis augmentation en fonction de la valeur du dollar canadien en 2011.

\section{Coûts directs}

Nous avons calculé les coûts associés à l'hospitalisation initiale à partir de l'étude de Dryden et collab. ${ }^{9}$ réalisée en Alberta.

Nous avons calculé le coût quotidien pour soins actifs liés aux hospitalisations suivant l'hospitalisation initiale en fonction des coûts établis par l'Initiative ontarienne de coût par cas $^{16}$, et ce, en incluant les hospitalisations de patients pour maladies du système génito-urinaire (47\%), maladies de la peau et des tissus sous-cutanés (35 \%) et maladies de l'appareil respiratoire $(18 \%)^{17}$. Cette approche a été choisie parce que la majorité des hospitalisations survenant après la blessure initiale sont associées à des complications concernant ces trois systèmes. Nous avons ensuite utilisé le coût de soins actifs ainsi déterminé pour attribuer une valeur à toutes les hospitalisations à court terme, y compris celles pour dépression, toxicomanie, etc.

Les professionnels de la santé non médecins consultés étaient des physiothérapeutes, des ergothérapeutes, des psychologues, des infirmières, des chiropraticiens, etc ${ }^{13}$. Les modifications apportées au domicile incluent tout ce qui avait été aménagé spécialement pour la personne atteinte d'un TME à son domicile principal et dans toute demeure secondaire ainsi qu'aux domiciles de sa famille et de ses amis ${ }^{14}$. Les articles sans ordonnance sont les analgésiques en vente libre, les cathéters, les pansements, les laxatifs, les vitamines et les gants en caoutchouc ${ }^{12}$. L'équipement adapté comprend les aides à la manipulation et à la respiration (p. ex. ventilateurs), les aides à la mobilité (p. ex. fauteuil roulant, orthèse et béquilles), les aides au sommeil et à l'hygiène (p. ex. lit d'hôpital, matelas spécial, soulève-lit ou siège élévateur de bain et fauteuil hygiénique) et les articles pour exercice ou autres (p. ex. vélo stationnaire, haltères, téléphone adapté) ${ }^{13}$.

Le coût horaire des soins auxiliaires a été déterminé selon le taux horaire canadien pour « autre personnel de soutien des services de santé ${ }^{18}$.

\section{Coûts indirects}

Nous avons suivi une approche du capital humain modifiée (selon laquelle la valeur du temps non rémunéré est mise en évidence) pour calculer les coûts indirects. De cette façon, l'année de vie ajustée en fonction de la qualité (AVAQ) a été estimée à 47834 \$ (taux salarial annuel moyen au Canada en 2011) ${ }^{19}$, sans tenir compte de l'âge de l'individu ou de sa situation relative à l'emploi. Le nombre d'années de vie perdues a été ajusté en fonction d'une qualité évaluée à 0,45 en matière d'utilité, soit le chiffre attribué aux personnes atteintes d'un $\mathrm{TME}^{20}$. Autrement dit, une année de vie d'une personne atteinte d'un TME équivaut à 0,45 AVAQ. La perte de 0,55 AVAQ a donc été évaluée à $26309 \$(0,55 \times 47834 \$)$. Les coûts indirects s'accumulent de ce fait chez les personnes atteintes d'un TME après la survenue de leur blessure.

Le nombre d'années de vie perdues chez les patients atteins de TME a été déterminé en fonction de l'âge et de l'espérance de vie estimée selon la gravité de la blessure à l'aide du rapport statistique annuel du National Spinal Cord Injury Statistical Center (NSCISC) de $2009^{21}$, et ajusté selon l'espérance de vie au sein de la population canadienne $^{22}$. Les personnes atteintes d'un TME décèdent plus tôt que si elles n'avaient pas subi leur blessure et le nombre d'années perdues augmente en fonction de la gravité de la blessure. Nous avons attribué une valeur de 47834 \$ à chacune de ces années de vie perdues.

Le fardeau économique lié aux TME au Canada a été calculé à l'aide d'une approche fondée sur l'incidence. "Une approche fondée sur l'incidence permet de mesurer les coûts (à vie) liés à toute nouvelle blessure survenant sur une période donnée (habituellement un an), alors qu'une approche fondée sur la prévalence permet de mesurer les coûts assumés par les personnes atteintes d'un TME pendant une période donnée de leur vie » [traduction] ${ }^{12, \text { p.14 }}$. Nous avons appliqué au nombre de patients atteints de TME survivant à l'hospitalisation au Canada par année (1 389) obtenu par Noonan et collab. ${ }^{23}$ la répartition des blessures selon leur gravité établie par Dryden et collab. ${ }^{10}: 106(7,7 \%)$ avec tétraplégie complète, 651 (46,8 \%) avec tétraplégie incomplète, $163(11,7 \%)$ avec paraplégie complète et 469 (33,8 \%) avec paraplégie incomplète.

Tous les coûts ont été actualisés à un taux annuel de $2 \%$. Le taux d'actualisation « est le taux auquel nous dévaluons les coûts survenant à l'avenir » [traduction] ${ }^{12,}$ p.168. Nous avons fait varier ce taux entre 0 et $4 \%$ dans l'analyse de sensibilité.

Les études économiques se servent souvent de l'analyse de sensibilité pour faire varier les hypothèses clés et déterminer si ces changements ont un effet important sur les résultats globaux. Pour la présente étude, nous avons appliqué des analyses de sensibilité à d'importants facteurs générateurs de coûts, notamment l'âge au moment de la blessure, le facteur de pondération relatif à l'incapacité, le taux d'actualisation, les hospitalisations, les consultations du fournisseur de soins de santé et les soins auxiliaires (tableau 1).

\section{Résultats}

Nous estimons que le fardeau économique à vie lié aux TME au Canada varie de 1,47 million de dollars chez les personnes atteintes d'une paraplégie incomplète à 3,03 millions de dollars pour les cas de 
TABLEAU 1

Hypothèses du modèle de base

\begin{tabular}{lcc} 
Domaine d'intérêt & Valeurs utilisées & $\begin{array}{c}\text { Analyse de } \\
\text { sensibilité }\end{array}$ \\
& Tétraplégie & Paraplégie \\
Complète Incomplète Complète Incomplète & \\
\hline
\end{tabular}

\section{Coûts directs}

Hospitalisation initiale (soins actifs et réadaptation)

$\begin{array}{lcccc}\text { DMS (en jours) } & 153,0 & 49,0 & 123,0 & 42,2 \\ \text { Coût (en dollars) } & 158049 & 46760 & 109418 & 42609 \\ \text { Nombre de services médicaux } & 53 & 16 & 38 & 15 \\ \text { Coût (en dollars) } & 10989 & 3156 & 7131 & 3551\end{array}$

Hospitalisations subséquentes, première année

Nombre moyen d'admissions

$\begin{array}{llll}0,30 & 0,31 & 0,26 & 0,23\end{array}$

DMS par admission (en jours)

10,1

8,6

8,7

5,2

Coût en soins actifs par jour (en dollars)

Coût (en dollars)

Hospitalisations subséquentes par année

$\begin{array}{lcccc}\text { Nombre moyen d'admissions } & 0,30 & 0,31 & 0,26 & 0,23 \\ \text { DMS par admission (en jours) } & 10,1 & 8,6 & 8,7 & 5,2 \\ \text { Coût en soins actifs par jour (en dollars) } & 1124 & 1124 & 1124 & 1124 \\ \text { Coût (en dollars) } & 3416 & 3036 & 2545 & 1321\end{array}$

3416

Services de professionnels de la santé par année

Nombre de visites chez le médecin

Nombre de visites chez des professionnels de la santé non médecins ${ }^{a}$

Coût par visite (en dollars)

Coût (en dollars)

Modifications apportées au domicile

Coût ponctuel (en dollars)

Domicile actuel

31149

29015

24540

25492

Autre domicile

9610

8912

9973

9701

Coûts annuels (en dollars)

Médicaments sur ordonnance

Produits en vente libre

Équipement adapté (moins de 5 ans) ${ }^{d}$

Équipement adapté (plus de 5 ans) ${ }^{d}$

Modifications apportées au véhicule

$\begin{array}{cccc}791 & 308 & 278 & 538 \\ 4867 & 4029 & 3376 & 2619 \\ 9724 & 6261 & 4890 & 3147 \\ 3695 & 2548 & 1578 & 885 \\ 1015 & 1015 & 1015 & 1015\end{array}$

Soins en établissement par année

Pourcentage de la population ayant un TME en établissement

Coût (en dollars)

10,1

1729,4

3,7

2,1

0,7

467,4

347,1

104,5

Soins auxiliaires par année

Heures par semaine

Heures par année

Pourcentage des heures payées ${ }^{e}$

Coût estimé par heure (en dollars)

Coût 'en dollars)

$\begin{array}{cccccl}56,3 & 31,9 & 13,5 & 16,9 & +/-25 \% & \text { Berkowitz et collab. }(1992)^{12} \\ 2928 & 1659 & 702 & 879 & & \text { Calculé } \\ 50 & 50 & 41 & 18 & & {\text { Berkowitz et collab. }(1992)^{12}}^{12} \\ 17,52 & 17,52 & 17,52 & 17,52 & +/-25 \% & \text { Statistique Canada }{ }^{18} \\ 51292 & 29062 & 12299 & 15397 & & \text { Calculé }\end{array}$

$+/-25 \%$

$+/-25 \%$

Dryden et collab. (2005) ${ }^{9}$

Dryden et collab. (2005) ${ }^{9}$

Dryden et collab. (2005) ${ }^{9}$

Dryden et collab. $(2005)^{9}$

Dryden et collab. (2004) ${ }^{11}$

Dryden et collab. (2004) ${ }^{11}$

Initiative ontarienne de coût par $\operatorname{cas}^{16}$

Calculé

Dryden et collab. (2004) ${ }^{11}$

Dryden et collab. (2004) ${ }^{11}$

Initiative ontarienne de coût par cas'

Calculé

+/- $25 \% \quad$ Harvey et collab. $(1992)^{13}$

$+/-25 \% \quad$ Harvey et collab. $(1992)^{13}$

+/- $25 \% \quad$ Calculé

Berkowitz et collab. (1992) ${ }^{12}$

Berkowitz et collab. (1998) ${ }^{14}$

Berkowitz et collab. (1998) ${ }^{14}$

Berkowitz et collab. (1992) ${ }^{12}$

Berkowitz et collab. (1992) ${ }^{12}$

Harvey et collab. $(1992)^{13}$

Harvey et collab. (1992) ${ }^{13}$

Berkowitz et collab. (1998) ${ }^{14}$

Berkowitz et collab. (1992) ${ }^{12}$

Berkowitz et collab. (1992) ${ }^{12}$

Suite page suivante 
TABLEAU 1 (Suite)

Hypothèses du modèle de base

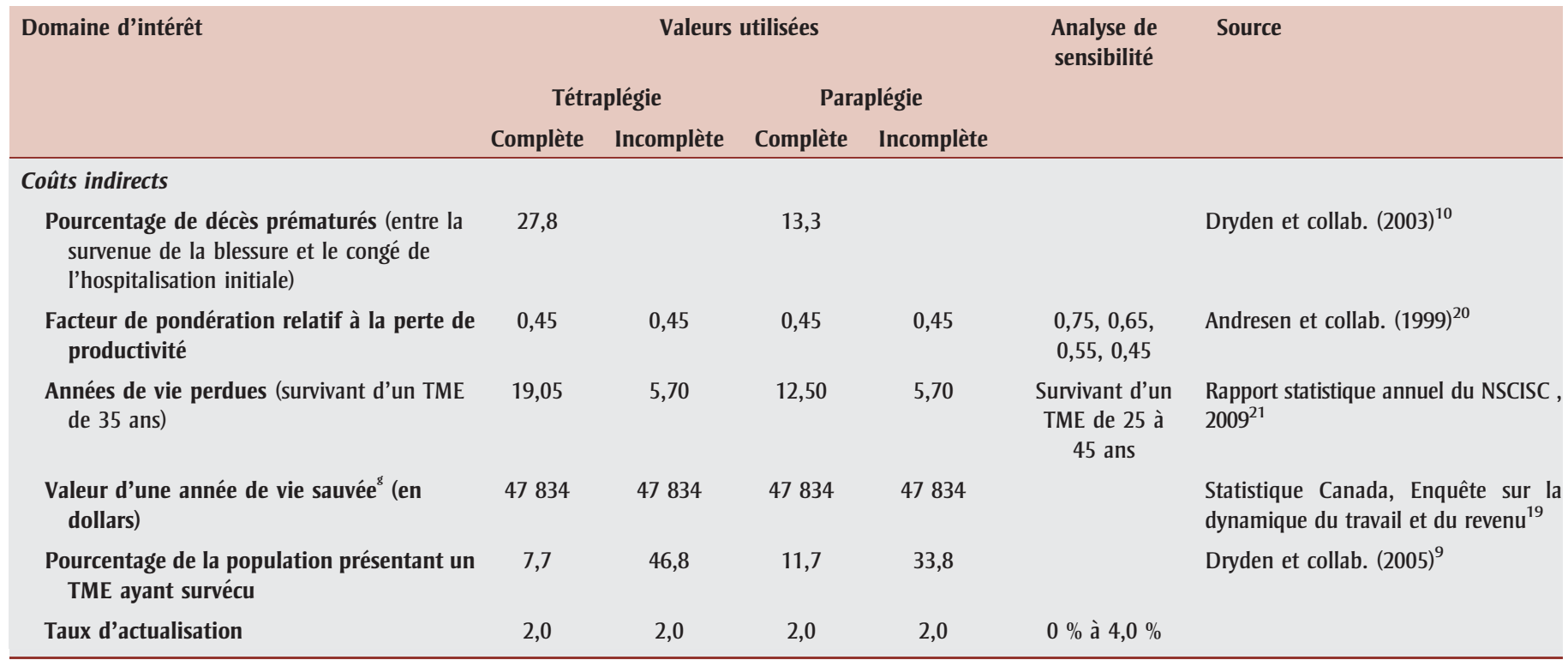

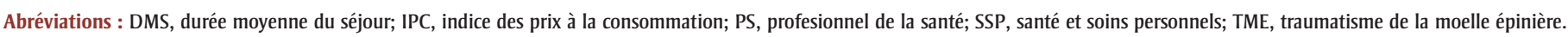

a Physiothérapeutes, ergothérapeutes, psychologues, infirmières, chiropraticiens, etc.

b Modifications apportées spécifiquement aux domiciles du survivant d'un TME ainsi qu'aux domiciles d'amis ou de membres de la famille.

c Analgésiques en vente libre, cathéters, pansements, laxatifs, vitamines et gants en caoutchouc, etc. ${ }^{12}$

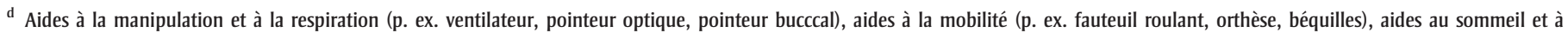
l'hygiène (p. ex. lit d’hôpital, matelas spécial, soulève-lit ou élévateur de bain, fauteuil hygiénique) et articles d'exercice ou autres (p. ex. vélo stationnaire, haltères, téléphone adapté).

e Proportion des heures de travail pour lesquelles le fournisseur de services a été rémunéré.

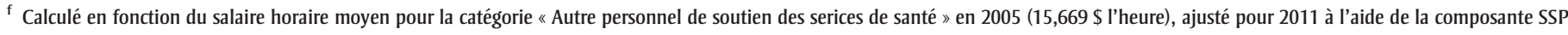
de I'IPC $(+11,8 \%)^{18}$.

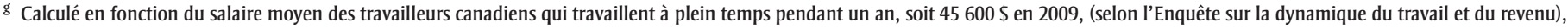
ajusté pour 2011 à l'aide de l'IPC $(+4,9 \%)^{19}$.

tétraplégie complète (tableau 2). Les coûts directs représentent de 44 à $51 \%$ des coûts totaux chez les patients paraplégiques, et de 56 à $66 \%$ des coûts totaux chez les patients tétraplégiques. Parmi les coûts directs, les plus importants facteurs générateurs de coûts étaient les soins auxiliaires offerts à la suite de la blessure. À eux seuls, ces coûts variaient entre 0,29 et 1,02 million de dollars (de 38 à $60 \%$ des coûts directs). Les frais d'hospitalisations variaient entre 0,08 et 0,23 million de dollars (de 5 à $13 \%$ des coûts directs) alors que les frais de consultation de professionnels de la santé variaient entre 0,07 et 0,15 million de dollars (de 10 à $13 \%$ des coûts directs).

Le fardeau économique annuel lié aux TME au Canada est estimé à 2,67 milliards de dollars (1,57 milliard de dollars en coûts directs et 1,10 milliard en coûts indirects, tableau 3). Les hospitalisations $(0,17$ milliard de dollars, soit $6,5 \%$ des coûts totaux), les consultations de professionnels de la santé $(0,18$ milliard de dollars; 6,7\% des coûts totaux), l'équipement et les modifications apportées au domicile (0,31 milliard de dollars; 11,6\% des coûts totaux) et les soins auxiliaires $(0,87$ milliard de dollars; $32,7 \%$ des coûts totaux) sont les plus importants facteurs générateurs de coûts.

Les résultats des analyses de sensibilité sont synthétisés dans le tableau 4. Les coûts sont sensibles en premier lieu au choix du taux d'actualisation. Si l'on ne tient pas compte de l'actualisation (taux effectif de $0 \%$ ), le fardeau économique à vie augmente de 38 à $47 \%$. Avec un taux d'actualisation de $4 \%$ (comparativement au taux de $2 \%$ utilisé comme référence) le fardeau économique à vie augmente de 23 à $27 \%$. L'âge auquel la blessure est survenue a également un effet important. Un âge moyen de 25 ans (comparativement à l'âge de référence de 35 ans) entraîne une augmentation des coûts globaux de 10 à $14 \%$, alors qu'un âge moyen de 45 ans entraîne une baisse de 14 à $18 \%$. Le fait de faire passer le facteur de pondération relatif à la perte de productivité de 0,45 pour toutes les blessures à 0,45 pour paraplégie incomplète, 0,55 pour tétraplégie incomplète, 0,65 pour paraplégie complète et 0,75 pour tétraplégie complète entraîne une augmentation des coûts indirects de 18 à $23 \%$.

Les coûts des soins directs sont sensibles en premier lieu aux hypothèses concernant le nombre d'heures en soins auxiliaires reçus. Une augmentation du nombre d'heures en soins reçus par année ou du taux de rémunération moyen de 25 \% entraîne une augmentation des coûts directs de 9 à $15 \%$. Si le nombre d'heures en soins et le taux de rémunération augmentent tous deux de $25 \%$, les coûts directs augmentent de 21 à $34 \%$. 
TABLEAU 2

Fardeau économique à vie lié aux traumatismes de la moelle épinière

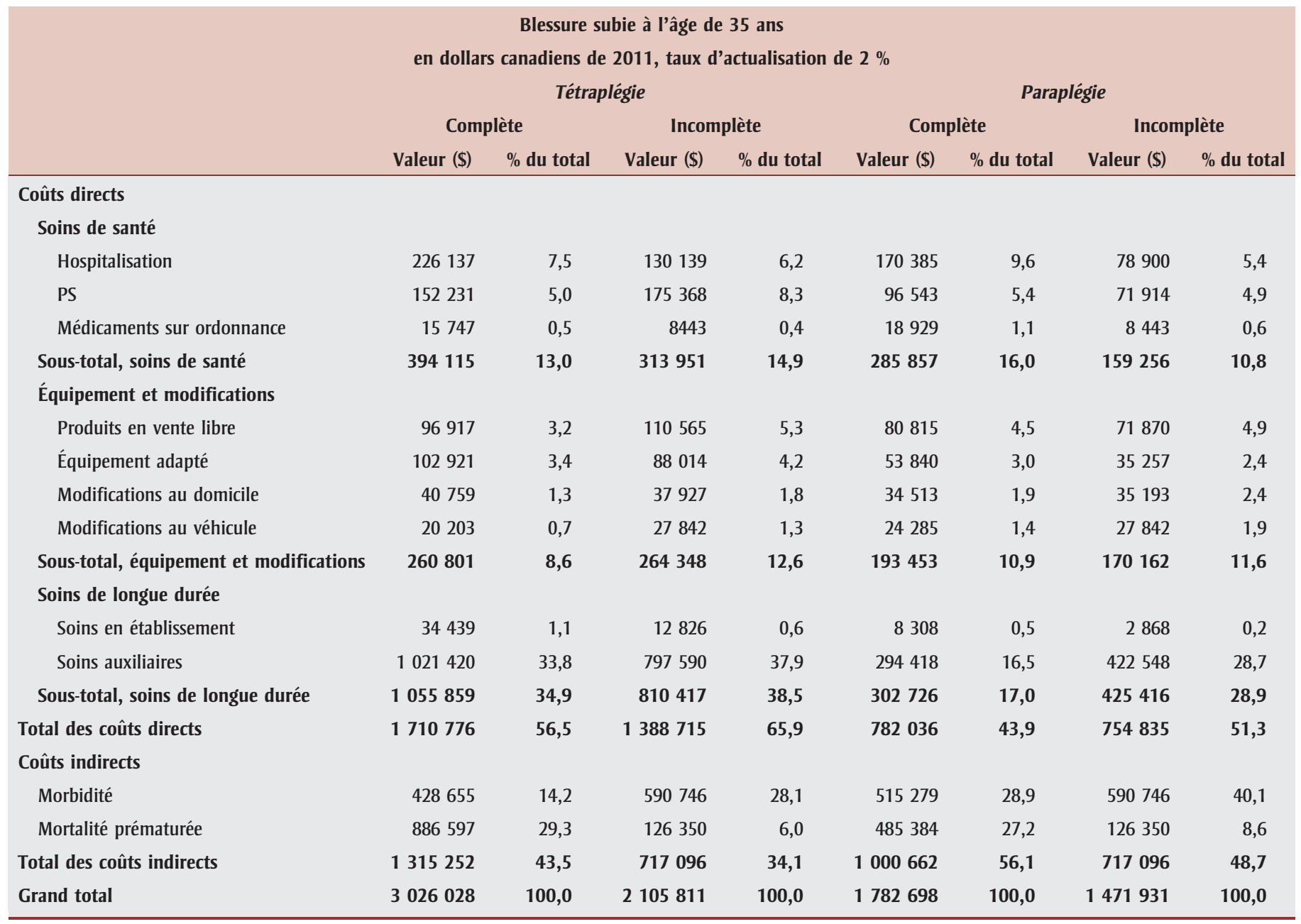

Abréviation : PS, professionnel de la santé.

\section{Analyse}

À notre connaissance, notre étude est la première tentative de quantification du fardeau économique à vie lié aux TME au Canada. Nous avons tenté d'être le plus exhaustifs possible dans l'inclusion des coûts.

La valeur des études sur les coûts des maladies a été remise en question, surtout $\mathrm{du}$ fait des approches diverses et des limites méthodologiques associées à ce genre de recherche $e^{24,25,26}$. Cependant, Segui-Gomez et Mackenzie ${ }^{27}$ soulignent l'importance de la variété des mesures, dont le fardeau économique, pour évaluer l'impact social des blessures, plus particulièrement des blessures non mortelles. Rice fait remarquer que les études sur les coûts de la maladie " expriment les effets indésirables des maladies et des blessures en dollars, ce qui est le langage universel des décideurs et des responsables des politiques. Ces estimations servent à : 1) définir en dollars l'ampleur de la maladie ou de la blessure; 2) justifier les programmes d'intervention; 3) faciliter l'affectation de fonds pour la recherche sur certaines maladies; 4) établir une base en matière de politiques et de planification en ce qui concerne les initiatives de prévention et de contrôle; 5) établir un cadre économique pour l'évaluation de

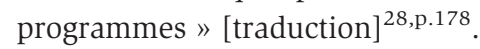

Malheureusement, il demeure difficile d'utiliser ces études pour élaborer des politiques. Par exemple, en 1995, le comité du Sénat américain sur les crédits a recommandé que les National Institutes of Health (NIH) préparent un rapport sur le coût social des maladies au sujet desquelles les $\mathrm{NIH}$ réalisent et financent de la recherche ${ }^{28}$. Une étude effectuée en 1998 par le U.S. Institute of Medicine recommandait aux $\mathrm{NIH}$ d'utiliser de façon plus rigoureuse les données sur « le fardeau de la maladie et les coûts connexes " au moment d'établir l'ordre des priorités en matière de financement de la recherche ${ }^{29}$. Malgré la production de rapports sur le coût de maladies spécifiques en 1995, en 1997 et en $2000^{28}$, il ne semble pas que ces données influencent l'affectation des fonds par les NIH pour les recherches en cours ${ }^{30,31}$.

Pour tenter de réduire l'hétérogénéité méthodologique des études sur les coûts de la maladie tout en reconnaissant la " forte demande soutenue en matière d'études d'impact économique » [traduction] $]^{32, p .2}$, des associations comme 
TABLEAU 3

Fardeau économique annuel lié aux traumatismes de la moelle épinière au Canada

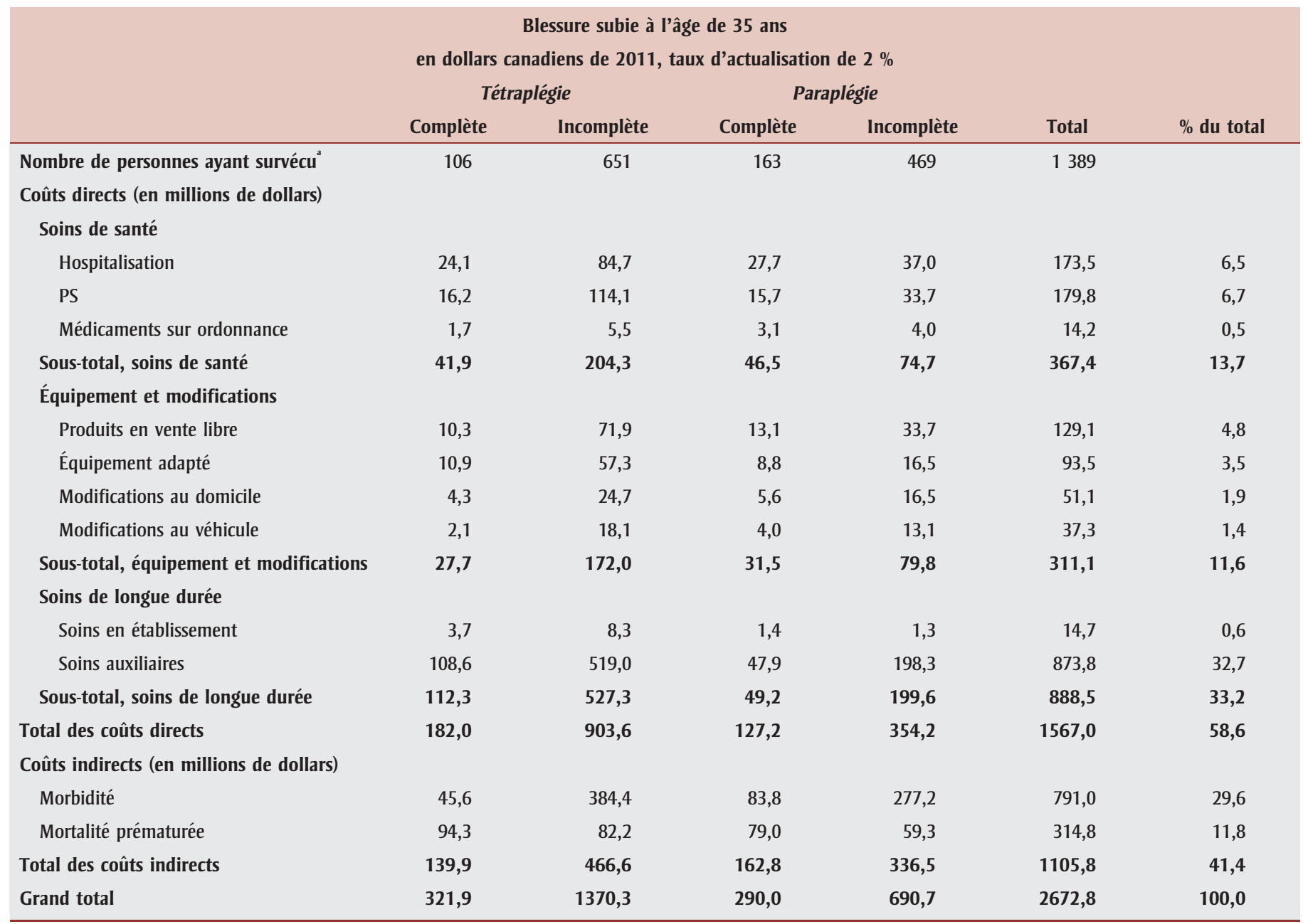

Abréviation : PS, professionnel de la santé.

a 1389 patients par année survivent à leur hospitalisation initiale ${ }^{23}$.

l'Organisation mondiale de la Santé ont proposé un cadre pour la tenue d'études sur les coûts de la maladie " ayant pour but d'accroître l'uniformité et la cohérence des études d'impact économique en santé » [traduction] $^{32, \text { p.3. }}$.

Cette analyse comporte d'importantes limites. Elle repose sur des données publiées provenant de diverses sources et périodes plutôt que sur une description détaillée des coûts d'une cohorte de patients. Les coûts moyens peuvent également cacher d'importantes variations dans les coûts individuels, même si l'on tient compte de la gravité de la blessure. Par conséquent, ces coûts ne devraient pas remplacer un plan de soins à vie préparé par des professionnels et les coûts de sa mise en œuvre. De plus, aucune tentative n'a été faite pour déterminer si ces coûts étaient associés à des soins optimaux ou même adéquats. Aux États-Unis, Webster et collab. ont constaté que "les personnes atteintes d'une tétraplégie à la suite d'un accident de travail peuvent se faire indemniser plus de frais médicaux après la première année suivant la blessure que les personnes qui n'ont pas droit à des avantages en matière d'indemnités d'accident de travail » [traduction] $]^{6, p .240}$.

Les coûts en soins de courte durée sont calculés à partir des coûts quotidiens globaux par patient atteint d'une maladie en particulier. Une autre approche consisterait à utiliser les pondérations du volume des ressources pour chaque hospitalisation et le coût estimé pour chacune de celles-ci. Cependant, les données nécessaires concernant des hospitalisations spécifiques ne sont pas disponibles à l'heure actuelle, problèmes que l'Institut Rick Hansen espère régler.

Bien qu'une méthode d'établissement des coûts fondée sur l'incidence repose en principe sur une trajectoire de coûts estimés à vie, elle nécessite en fait une hypothèse (pour cette étude) quant à l'état fonctionnel à un moment donné. Pour déterminer les changements réels en ce qui a trait aux coûts liés à un changement d'état fonctionnel, il faut absolument avoir accès à des données à long terme pour chaque patient.

La méthode la plus souvent utilisée pour déterminer les coûts indirects est 
TABLEAU 4

Fardeau économique à vie lié aux traumatismes de la moelle épinière

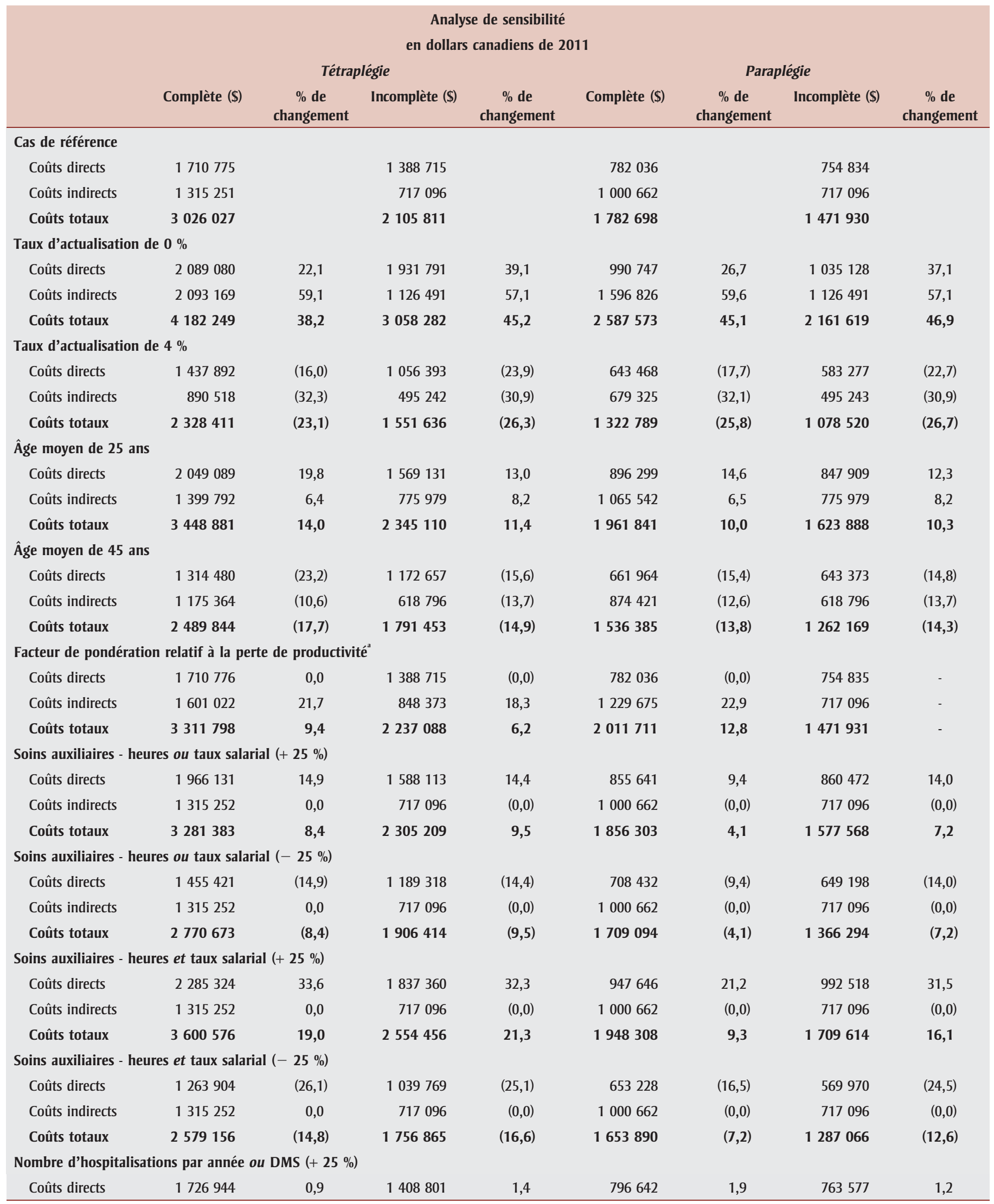


TABLEAU 4 (Suite)

Fardeau économique à vie lié aux traumatismes de la moelle épinière

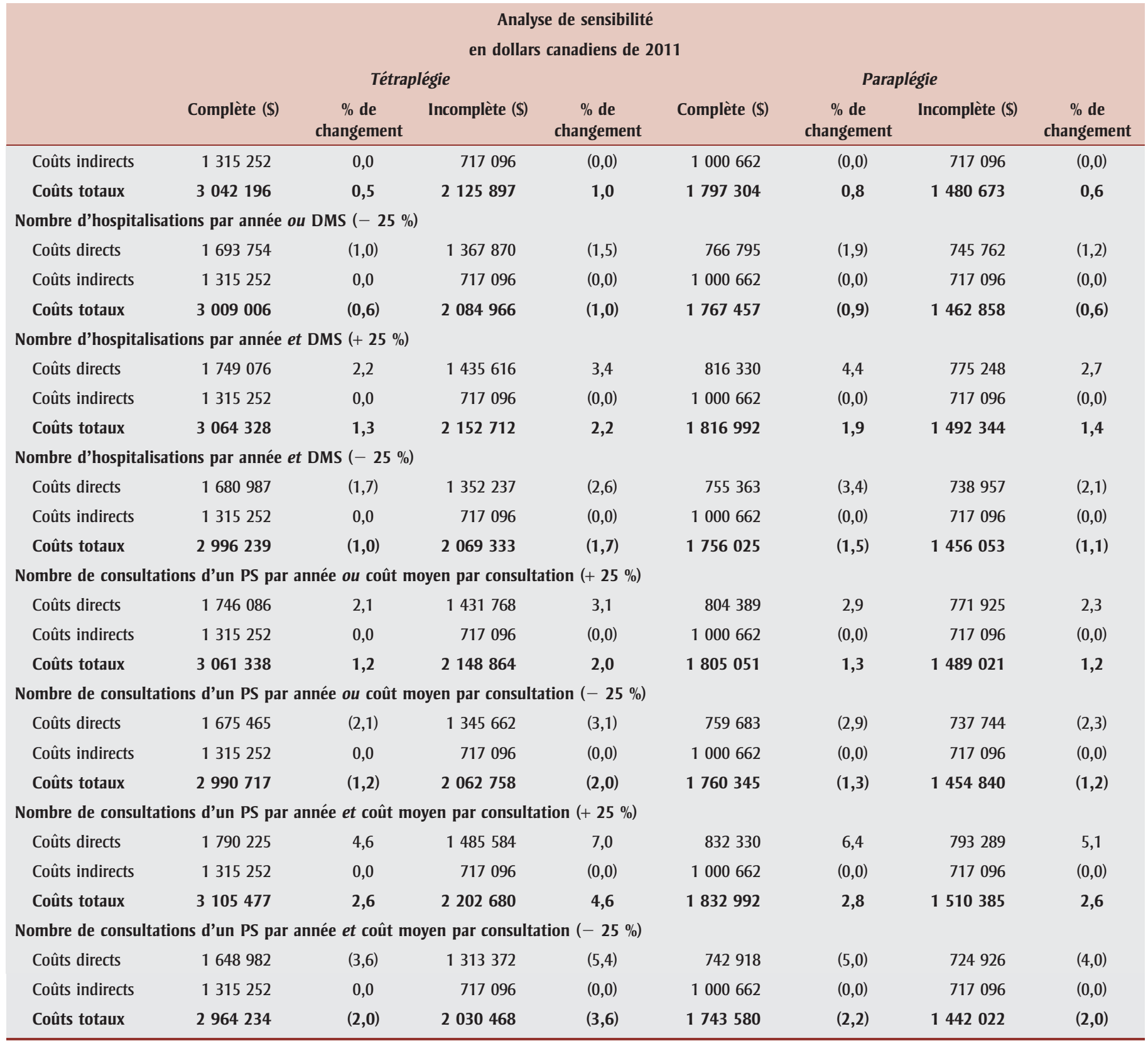

Abréviations : DMS, durée moyenne du séjour; PS, professionnel de la santé.

a Tétraplégie complète : 0,75; paraplégie complète : 0,65; tétraplégie incomplète : 0,55; paraplégie incomplète : 0,45.

l'approche du capital humain, qui consiste à combiner les revenus moyens selon le sexe et l'âge avec les tendances en matière de productivité et les années de vie perdues en raison d'une maladie spécifique pour estimer les revenus non réalisés à vie. Une importante critique de cette méthode provient du fait qu'elle accorde plus de valeur aux années de vie perdues par des personnes ayant un potentiel de revenu plus élevé (p. ex. hommes de 35 à 55 ans) que celles ayant un potentiel moins élevé (p. ex. femmes de 75 ans et plus $)^{33}$. Le travail non rémunéré et le temps de loisirs ne sont de fait pas expressément pris en compte dans l'approche du capital humain ${ }^{34}$.

Pour le calcul des coûts indirects, nous avons suivi une approche du capital humain modifiée, selon laquelle on attribue une valeur explicite au temps non rémunéré. Cette approche a consisté à attribuer une valeur de $47 \quad 834 \quad$ \$ (salaire annuel moyen au Canada en 2011) à chaque AVAQ perdue. Plusieurs chercheurs ont suggéré d'attribuer une valeur au temps non rémunéré dans l'approche du capital humain ${ }^{35,36}$ pour contrer la tendance inhérente de cette approche à sous-évaluer l'impact d'une maladie ou d'une blessure chez les personnes âgées à la retraite, les personnes handicapées et les personnes qui choisissent de ne pas exercer d'emploi rémunéré. 
Cao et collab. ${ }^{4}$ ont récemment estimé les coûts directs (par opposition aux frais) à vie moyens aux États-Unis pour une blessure survenue à l'âge de 25 ans et selon un taux d'actualisation de $2 \%$. Ces coûts variaient de 1,10 million de dollars (dollars US en 2009) pour une blessure de type D selon l'échelle de déficience de l'American Spinal Injury Association (ASIA) à n'importe quel niveau à 3,41 millions de dollars pour une blessure de type $\mathrm{A}, \mathrm{B}$ ou $\mathrm{C}$ au niveau (vertèbre) $\mathrm{C} 1$ à $\mathrm{C} 4^{4}$. En utilisant les mêmes grandes hypothèses (blessure survenue à l'âge de 25 ans, taux d'actualisation de $2 \%$ ), nous arrivons à une valeur actuelle des coûts directs moyens à vie qui varie de 0,85 à 2,05 milliards de dollars, soit d'environ 23 à $40 \%$ moins élevée que l'estimation pour les États-Unis.

Cette différence peut être attribuable au coût global plus élevé des prestations de soins de santé aux États-Unis, ce qui s'explique par une combinaison de prix plus élevés, des coûts administratifs indirects, l'utilisation d'équipement très coûteux et la pratique d'une " médecine défensive » en raison du régime juridique de responsabilité délictuelle propre aux États-Unis. Il est estimé que les frais excédentaires aux États-Unis sont environ $40 \%$ plus élevés que ceux des pays étrangers utilisés comme points de comparaison $^{38}$.

La différence peut également être attribuable à la façon de quantifier les soins auxiliaires. DeVivo et collab. ${ }^{5}$ ont estimé que les heures annuelles en soins auxiliaires à partir de la deuxième année suivant la blessure s'élevaient à 1124 pour une blessure de type $\mathrm{D}$ selon l'échelle de déficience de l'ASIA à n'importe quel niveau, et à 5453 pour les blessures de type $\mathrm{A}, \mathrm{B}$ ou $\mathrm{C}$ au niveau $\mathrm{C} 1-\mathrm{C} 4^{5}$. Chaque heure, rémunérée ou non, a été évaluée à 21,00 \$ (dollars US en 2009). Le nombre d'heures par année semble avoir été déterminé " à partir d'auto-déclarations de toutes les personnes figurant dans la base de données du National Spinal Cord Injury Statistical Center (NSCISC) qui ont rempli une évaluation de suivi annuel entre 2000 et 2006 et fourni toutes les données demandées pour ce point " [traduction] $]^{5, p .4}$. Nous avons utilisé une estimation beaucoup plus basse (de 702 à 2928 heures par année), celle de l'étude basée sur la population de Berkowitz et collab. ${ }^{14}$, et nous avons appliqué le tarif de 17,52 \$ de l'heure aux heures rémunérées et non rémunérées. Des estimations détaillées des coûts de soins auxiliaires au sein de la population canadienne atteinte d'un TME sont nécessaires, en grande partie parce que ces coûts représentent le plus grand facteur générateur de coûts directs.

En dernier lieu, il faut souligner la différence entre l'utilisation de la prévalence dans les établissements plutôt que la prévalence dans la population. Dans les études fondées sur la population menées au Canada et aux États-Unis, la proportion de personnes atteintes d'un TME avec tétraplégie complète ou paraplégie complète est similaire au sein de la population, soit respectivement de 7 à $8 \%$ et de 10 à $12 \%$, alors que la prévalence de tétraplégie complète ou de paraplégie complète chez les personnes atteintes d'un TME recevant des soins offerts par le U.S. Model System a tendance à être beaucoup plus élevée, soit respectivement de $24 \%$ et de $27 \%{ }^{10,12}$. Il est important de tenir compte de cette surpondération des blessures plus graves, surtout dans l'estimation du fardeau économique annuel lié aux TME.

\section{Conclusion}

À notre connaissance, cette étude représente la première tentative de quantification du fardeau économique lié aux TME au Canada. Malgré le nombre relativement faible de blessures par année, le fardeau économique annuel, soit 2,67 milliards de dollars, est très lourd. Il est possible d'alléger ce fardeau en prévenant certains nouveaux cas, en améliorant les capacités des patients ${ }^{39}$ ou encore en évitant les complications secondaires (par des améliorations fonctionnelles ou par une meilleure gestion) après la survenue de la blessure.

\section{Remerciements}

L'étude a été financée par l'institut Rick Hansen et Santé Canada.
Hans Krueger et Logan Trenaman ont reçu du financement de l'institut Rick Hansen pour effectuer leur travail. Vanessa Noonan, Phalgun Joshi et Carly Rivers travaillent à l'Institut Rick Hansen.

\section{Références}

1. French DD, Campbell RR, Sabharwal S, Nelson AL, Palacios PA, Gavin-Dreschnack D. Health care costs for patients with chronic spinal cord injury in the Veterans Health Administration. J Spinal Cord Med. 2007;30(5):477.

2. St Andre JR, Smith BM, Stroupe KT, Burns SP, Evans CT, Ripley DC et collab. A comparison of costs and health care utilization for veterans with traumatic and nontraumatic spinal cord injury. Top Spinal Cord Inj Rehabil. 2011;16(4):27-42.

3. DeVivo MJ. Causes and costs of spinal cord injury in the United States. Spinal Cord. 1997;35(12):809-13.

4. Cao Y, Chen Y, DeVivo M. Lifetime direct costs after spinal cord injury. Top Spinal Cord Inj Rehabil. 2011;16(4):10-6.

5. DeVivo M, Chen Y, Mennemeyer ST, Deutsch A. Cost of care following spinal cord injury. Top Spinal Cord Inj Rehabil. 2011;16(4):1-9.

6. Webster B, Giunti G, Young A, Pransky G, Nesathurai S. Work-related tetraplegia: cause of injury and annual medical costs. Spinal Cord. 2004;42(4):240-7.

7. Kiekens C, Van Rie K, Peers K, Lysens R. Cost of rehabilitation care in traumatic and nontraumatic spinal cord injury in a European context. Top Spinal Cord Inj Rehabil. 2011;16(4):43-52.

8. DeVivo M, Farris V. Causes and costs of unplanned hospitalizations among persons with spinal cord injury. Top Spinal Cord Inj Rehabil. 2011;16(4):53-61.

9. Dryden DM, Saunders LD, Jacobs $\mathrm{P}$ et collab. Direct health care costs after traumatic spinal cord injury. J Trauma. 2005; $59(2): 443-9$

10. Dryden DM, Saunders LD, Rowe BH et collab. The epidemiology of traumatic spinal cord injury in Alberta, Canada. Can J Neurol Sci. 2003;30(2):113-21. 
11. Dryden DM, Saunders LD, Rowe BH et collab. Utilization of health services following spinal cord injury: a 6-year follow-up study. Spinal Cord. 2004;42(9):513-25.

12. Berkowitz M, Harvey C, Greene CG, Wilson $\mathrm{SE}$. The economic consequences of traumatic spinal cord injury. New York: Demos Medical Publishing; 1992.

13. Harvey C, Wilson SE, Greene CG, Berkowitz M, Stripling TE. New estimates of the direct costs of traumatic spinal cord injuries: results of a nationwide survey. Paraplegia. 1992;30(12):834-50.

14. Berkowitz M, O'Leary PK, Kruse DL, Harvey C. Spinal cord injury: an analysis of medical and social costs. New York: Demos Medical Publishing; 1998.

15. Statistique Canada. Indice des prix à la consommation (IPC) [Internet]. Ottawa (Ont.) : Statistique Canada; 2010 [consulté le 15 novembre 2011]. Consultable à la page : http://www23.statcan.gc.ca/imdb $/$ p2SV_f.pl?Function = getSurvey\&SDDS $=2301$ \&lang $=\mathrm{en} \& \mathrm{db}=\mathrm{imdb} \& \mathrm{adm}=8 \& \mathrm{dis}=2$

16. Initiative ontarienne de coût par cas. Outil d'analyse des coûts [Internet]. Toronto (Ont.) : Initiative ontarienne de coût par cas; 2011 [consulté le 15 novembre 2011]. Consultable à partir de la page : www.occp.com/mainPage.htm

17. Cardenas DD, Hoffman JM, Kirshblum S, McKinley W. Etiology and incidence of rehospitalization after traumatic spinal cord injury: a multicenter analysis. Arch Phys Med Rehabil. 2004;85(11):1757-63.

18. Statistique Canada. Gains médians(1) et emploi, travailleurs à plein temps toute l'année, toutes les professions, les deux sexes, pour le Canada, les provinces et les territoires - Données-échantillon (20 \%) [Internet]. Ottawa (Ont.) : Statistique Canada; 2010 [consulté le 15 novembre 2011]. Consultable à la page : http://www12 statcan.gc.ca/census-recensement/2006/dp-pd /hlt/97-563/T801-fra.cfm?Lang $=$ F\&T $=801 \&$ $\mathrm{GH}=4 \& \mathrm{GF}=0 \& \mathrm{G} 5=0 \& \mathrm{SC}=1 \& \mathrm{SR}=1 \& \mathrm{SO}$ $=99 \& \mathrm{O}=\mathrm{A} \& \mathrm{D} 1=1 \& \mathrm{D} 2=1$

19. Statistique Canada. Le revenu au Canada [Internet]. Ottawa (Ont.) : Statistique Canada, 2011 [consulté le 25 novembre 2011]. Consultable à la page : http: //www.statcan.gc.ca/pub/75-202-x/75-202 -x2009000-fra.htm
20. Andresen EM, Fouts BS, Romeis JC, Brownson CA. Performance of healthrelated quality-of-life instruments in a spinal cord injured population. Arch Phys Med Rehabil. 1999;80(8):877-84

21. National Spinal Cord Injury Statistical Center (NSCISC). NSCISC annual statistical report [Internet]. Birmingham (AL): NSCISC; 2009 [consulté le 15 novembre 2011]. PDF téléchargeable à partir du lien : https://www .nscisc.uab.edu/PublicDocuments/reports /pdf/2009\%20NSCISC \%20Annual \%20 Statistical\%20Report \%20-\%20Complete\% 20Public\%20Version.pdf

22. Statistique Canada. Tables de mortalité, Canada, provinces et territoires [Internet]. Ottawa (Ont.) : Statistique Canada; 2006 [consulté le 15 novembre 2011]. Consultable à la page : http://www5.statcan.gc.ca /bsolc/olc-cel/olc-cel?catno $=84-537-$ XIF\& lang $=$ fra

23. Noonan VK, Fingas M, Farry A et collab. Incidence and prevalence of spinal cord injury in Canada: a national perspective. Neuroepidemiology. 2012;38(4):219-26.

24. Currie G, Kerfoot KD, Donaldson C, Macarthur C. Are cost of injury studies useful? Inj Prev. 2000 Sep;6(3):175-6.

25. Bloom BS, Bruno DJ, Maman DY, Jayadevappa R. Usefulness of US cost-ofillness studies in healthcare decision making. Pharmacoeconomics. 2001;19(2):207-13.

26. Clabaugh G, Ward MM. Cost-of-illness studies in the United States: a systematic review of methodologies used for direct cost. Value Health. 2008 Jan-Feb;11(1):13-21.

27. Segui-Gomez M, MacKenzie EJ. Measuring the public health impact of injuries. Epidemiol Rev. 2003;25:3-19.

28. Rice DP. Cost of illness studies: what is good about them? Inj Prev. 2000 Sep;6(3):177-9.

29. Institute of Medicine (US) Committee on the NIH Research Priority-Setting Process. Scientific opportunities and public needs: improving priority setting and public input at the National Institutes of Health. Washington (DC): National Academies Press; 1998.
30. Gross CP, Anderson GF, Powe NR. The relation between funding by the National Institutes of Health and the burden of disease. N Engl J Med. 1999 Jun 17;340(24):1881-7.

31. Gillum LA, Gouveia C, Dorsey ER et collab. NIH disease funding levels and burden of disease. PLoS One. 2011 Feb 24;6(2):e16837.

32. World Health Organization, Department of Health Systems. WHO guide to identifying the economic consequences of disease and injury. Geneva (CH): World Health Organization; 2009.

33. Yabroff KR, Bradley $\mathrm{CJ}$, Mariotto $\mathrm{AB}$, Brown ML, Feuer EJ. Estimates and projections of value of life lost from cancer deaths in the United States. J Natl Cancer Inst. 2008 Dec 17;100(24):1755-62.

34. Tranmer JE, Guerriere DN, Ungar WJ, Coyte PC. Valuing patient and caregiver time: a review of the literature. Pharmacoeconomics. 2005;23(5):449-59.

35. Santé Canada, Division de la recherche sur les politiques. Le fardeau économique de la maladie au Canada, 1998 [Internet]. Ottawa (Ont.) : Santé Canada; 1998 [consulté le 15 novembre 2011]. PDF (2,31 Mo) téléchargeable à partir du lien : http://www.phac-aspc .gc.ca/ebic-femc/ebic-femc98/pdf/femc1998 .pdf

36. Krueger H, Lindsay P, Cote R, Kapral MK, Kaczorowski J, Hill MD. Cost avoidance associated with optimal stroke care in Canada. Stroke. 2012 Aug;43(8):2198-206.

37. Reinhardt UE. Why does U.S. health care cost so much? (Part I) [Internet]. New York (NY): New York Times; 2008 [consulté le 15 novembre 2011]. Consultable à la page : http://economix.blogs.nytimes.com/2008 /11/14/why-does-us-health-care-cost-so-much -part-i/

38. Anderson GF, Reinhardt UE, Hussey PS, Petrosyan V. It's the prices, stupid: why the United States is so different from other countries. Health Aff. 2003;22(3):89-105.

39. Fehlings MG, Vaccaro A, Wilson JR et collab. Early versus delayed decompression for traumatic cervical spinal cord injury: results of the Surgical Timing in Acute Spinal Cord Injury Study (STASCIS). PloS One. 2012;7(2):e32037. 